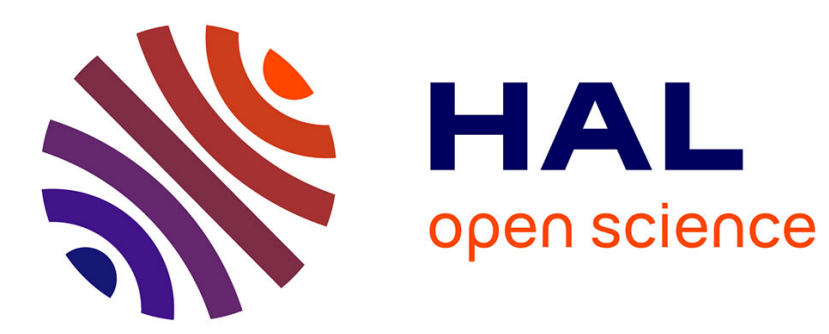

\title{
La responsabilité sociale de l'entreprise et les accords volontaires sont-ils complémentaires?
}

Pierre Fleckinger, Matthieu Glachant

\section{To cite this version:}

Pierre Fleckinger, Matthieu Glachant. La responsabilité sociale de l'entreprise et les accords volontaires sont-ils complémentaires?. Economie et Prévision, 2009, 4-5 (190-191), pp.95-105. hal00447028

HAL Id: hal-00447028

https://hal-mines-paristech.archives-ouvertes.fr/hal-00447028

Submitted on 14 Jan 2010

HAL is a multi-disciplinary open access archive for the deposit and dissemination of scientific research documents, whether they are published or not. The documents may come from teaching and research institutions in France or abroad, or from public or private research centers.
L'archive ouverte pluridisciplinaire HAL, est destinée au dépôt et à la diffusion de documents scientifiques de niveau recherche, publiés ou non, émanant des établissements d'enseignement et de recherche français ou étrangers, des laboratoires publics ou privés. 


\title{
La Responsabilité Sociale de l'Entreprise et les Accords Volontaires sont-ils complémentaires ?*
}

\author{
Pierre Fleckinger, Matthieu Glachant ${ }^{\ddagger}$
}

\begin{abstract}
Résumé
En une vingtaine d'années, les Accords Volontaires (AV, dans la suite du texte) sont devenus des outils classiques de la politique environnementale. Ce sont des dispositifs dans lesquels une firme, ou un groupe de firmes, s'engage volontairement devant une autorité de régulation à atteindre un objectif environnemental allant au delà des exigences réglementaires. En Europe ou au Japon, les AV sont le plus souvent des accords négociés au cours desquels les firmes et le régulateur déterminent par la négociation les engagements. La Commission Européenne a ainsi passé des accords avec les associations représentant les constructeurs automobiles européens (ACEA), japonais (JAMA) et coréens (KAMA) pour réduire les émissions de $\mathrm{CO} 2$ des nouveaux véhicules. Aux Etats-Unis, la forme habituelle des AV est le programme volontaire (voluntary program). A la différence des accords négociés, les objectifs environnementaux sont prédéfinis par le régulateur. Au 1er juillet 2009, le site internet de l'agence de l'environnement américaine (EPA) recensait 62 programmes volontaires dans différents secteurs.

Les AV traditionnels sont bilatéraux par nature puisqu'ils associent des entreprises et le régulateur. Si les engagements des premières sont clairs - atteindre un objectif environnemental, ceux du régulateur le sont beaucoup moins. Cependant, on peut penser qu'il s'engage implicitement à suspendre l'intervention publique dans les domaines couverts par l'accord. Sous cette hypothèse, les AV sont donc obtenus sous la menace d'une action législative ou réglementaire.
\end{abstract}

${ }^{*}$ Nous tenons à remercier l'éditeur, Pierre Malgrange, deux rapporteurs anonymes pour leurs précieux conseils, ainsi que de nombreux participants à différents séminaires et conférences.

${ }^{\dagger} \mathrm{CES}$, Université Paris 1 Panthéon-Sorbonne. Email : pierre.fleckinger@univ-paris1.fr.

‡CERNA, Mines ParisTech. Email : glachant@ensmp.fr. 
Depuis l'introduction des $\mathrm{AV}$, la tendance générale est à un rôle plus actif des firmes. Avec le développement du concept de "Responsabilité Sociale de l'Entreprise" (RSE), les firmes vont même jusqu'à s'auto-réguler avant l'implication de toute autorité publique. Contrairement aux AV, la RSE est purement unilatérale en ce sens qu'elle n'est liée à aucune promesse, même implicite, de la part du régulateur. Nous mettrons cette différence entre la RSE et les AV au coeur de notre analyse. Le développement de la RSE suscite des interrogations sur la motivation des firmes la pratiquant. Entreprennent-elles ces activités pour améliorer leur réputation? S'engagentelles pour des raisons de marché liées à l'existence de consommateurs verts? Ou encore, la RSE permet-elle d'influencer les réglementations à venir, voire de les éviter? Dans cet article nous concentrons l'analyse sur les activités de RSE motivées par la volonté de préempter ou d'influencer le contenu des futures politiques publiques.

En adoptant le point de vue du régulateur, nous cherchons à répondre aux questions suivantes : quel est le rôle des AV aujourd'hui dans un contexte où les firmes s'engagent dans la RSE? RSE et AV sont-ils des substituts ou des compléments du point de vue de la puissance publique? Quelle est l'influence de la RSE sur le bienêtre social atteignable avec un AV?

Pour répondre à ces questions, nous construisons un modèle simple d'élaboration d'une politique publique mettant en scène une firme et un régulateur. La firme peut dans un premier temps s'engager sur un niveau de RSE, c'est-à-dire s'engager à améliorer sa performance sociale ou environnementale. Ainsi nous nous intéressons à la RSE en tant qu'engagement crédible de la firme, prenant par exemple la forme d'investissements spécifiques irréversibles. Dans la seconde étape du jeu, le régulateur définit sa stratégie d'intervention. Il dispose de deux options : engager directement un processus législatif imposant un quota d'émission ou négocier un accord volontaire avec la firme.

Une importante littérature théorique développe des analyses des effets des AV, de la RSE ou de l'auto-régulation sur le bien-être social. On peut classer ces travaux en deux catégories. La première rassemble les contributions dans lesquelles les firmes s'engagent dans des activités environnementales ou sociales pour des raisons de marché. Dans la seconde, elles le font pour éviter une intervention publique ou pour en influencer le contenu. Notre contribution se rattache à cette seconde catégorie.

Notre travail est en cela proche de celui de Lyon et Maxwell (2003). La séquence décrite dans leur modèle est la suivante. Après une étape de RSE, le régulateur initie un processus législatif, qui aboutit à un quota avec une certaine probabilité fixe. En dernière étape, un AV est mis en place si la tentative de légiférer n'a pas abouti. Leur hypothèse clef est que l'AV est associé à une subvention qui fournit à la firme 
les incitations à s'engager. La première étape de notre modèle est identique mais les deux suivantes sont inversées : le régulateur tente d'obtenir un accord volontaire à la deuxième étape et ne s'engage dans la voie législative qu'à la troisième étape si la firme refuse l'accord. La firme s'engage donc pour éviter ou atténuer le quota déterminé par voie législative. Notre séquence nous semble plus réaliste puisque dans la pratique, les $\mathrm{AV}$ ne conduisent pas à des transferts monétaires vers les entreprises - ce dont conviennent d'ailleurs Lyon et Maxwell (2003). A partir du moment où l'AV n'est pas associé à une subvention, la séquence que nous modélisons - négocier d'abord et légiférer en cas de désaccord - est la seule permettant l'émergence d'un accord à l'équilibre.

Nous montrons que l'effort de RSE réduit le bien-être social par rapport à un scénario sans RSE quand la séquence est RSE AV législation. En revanche, nous montrons que la RSE augmente le bien être social en l'absence d'AV, c'est-à-dire quand la séquence se limite à : RSE législation. La RSE et les AV ne sont donc pas complémentaires dans l'optique d'un régulateur. Faut-il alors renoncer aux accords volontaires quand les firmes font déjà de la RSE ? La réponse est en fait ambiguë et dépend de la répartition du pouvoir de négociation entre la firme et le régulateur. En présence de RSE, les AV ne restent utiles que quand le régulateur dispose d'un fort pouvoir de négociation. Ainsi, l'usage de solutions négociées dans les politiques publiques, s'il apparaît Pareto-améliorant tant que le comportement des entreprises est fixé, s'avère moins efficace, voire néfaste, quand les entreprises peuvent préempter ces négociations. 


\section{Introduction}

En une vingtaine d'années, les Accords Volontaires (AV, dans la suite du texte) sont devenus des outils classiques de la politique environnementale. ${ }^{1}$ Ce sont des dispositifs dans lesquels une firme, ou un groupe de firmes, s'engage volontairement devant une autorité de régulation à atteindre un objectif environnemental allant au delà des exigences réglementaires. En Europe ou au Japon, les AV sont le plus souvent des accords négociés au cours desquels les firmes et le régulateur déterminent par la négociation les engagements. La Commission Européenne a par exemple passé des accords avec les associations représentant les constructeurs automobiles européens (ACEA), japonais (JAMA) et coréens (KAMA) pour réduire les émissions de $\mathrm{CO} 2$ des nouveaux véhicules. Aux Etats-Unis, la forme habituelle des AV est le programme volontaire (voluntary program). A la différence des accords négociés, les objectifs environnementaux sont prédéfinis par le régulateur. $\mathrm{Au}$ 1er juillet 2009, le site internet de l'agence de l'environnement américaine (EPA) recensait 62 programmes volontaires dans différents secteurs.

Les AV traditionnels sont bilatéraux par nature puisqu'ils associent des entreprises et le régulateur. Si les engagements des premières sont clairs - atteindre un objectif environnemental -, ceux du régulateur le sont beaucoup moins. Cependant, on peut penser qu'il s'engage implicitement à suspendre l'intervention publique dans les domaines couverts par l'accord. Sous cette hypothèse, les AV sont donc obtenus sous la menace d'une action législative ou réglementaire.

Depuis l'introduction des $\mathrm{AV}$, la tendance générale est à un rôle plus actif des firmes. Avec le développement du concept de "Responsabilité Sociale de l'Entreprise" (RSE), les firmes vont même jusqu'à s'auto-réguler avant l'implication de toute autorité publique. Contrairement aux AV, la RSE est purement unilatérale en ce sens qu'elle n'est liée à aucune promesse, même implicite, de la part du régulateur. Nous mettrons cette différence entre la RSE et les AV au coeur de notre analyse. Le développement de la RSE suscite des interrogations sur la motivation des firmes la pratiquant. Entreprennent-elles ces activités pour améliorer leur réputation? S'engagent-elles pour des raisons de marché liées à l'existence de consommateurs verts? Ou encore, la RSE permet-elle d'influencer les réglementations à venir, voire de les éviter? Nous ne traitons pas ces questions dans cet article. Nous concentrons directement l'analyse sur les activités de RSE motivées par la volonté de préempter ou d'influencer le contenu des futures politiques publiques.

En adoptant le point de vue du régulateur, nous cherchons à répondre aux questions suivantes : quel est le rôle des $\mathrm{AV}$ aujourd'hui dans un contexte où les firmes s'engagent 
dans la RSE ? RSE et AV sont-ils des substituts ou des compléments du point de vue de la puissance publique? Quelle est l'influence de la RSE sur le bien-être social atteignable avec un AV?

Pour répondre à ces questions, nous construisons un modèle simple d'élaboration d'une politique publique mettant en scène une firme et un régulateur. La firme peut dans un premier temps s'engager sur un niveau de RSE, c'est-à-dire s'engager à améliorer sa performance sociale ou environnementale. Ainsi nous nous intéressons à la RSE en tant qu'engagement crédible de la firme, prenant par exemple la forme d'investissements spécifiques irréversibles. ${ }^{2}$ Dans la seconde étape du jeu, le régulateur définit sa stratégie d'intervention. Il dispose de deux options : engager directement un processus législatif imposant un quota d'émission ou négocier un accord volontaire avec la firme.

Une importante littérature théorique développe des analyses des effets des $\mathrm{AV}$, de la RSE ou de l'auto-régulation sur le bien-être social. ${ }^{3}$ On peut classer ces travaux en deux catégories. La première rassemble les contributions dans lesquelles les firmes s'engagent dans des activités environnementales ou sociales pour des raisons de marché. Dans la seconde, elles le font pour éviter une intervention publique ou pour en influencer le contenu.

Bien que notre contribution relève de la seconde catégorie, il n'est pas inutile de présenter brièvement le premier courant de recherche. Son point de départ est le constat que certains agents économiques-consommateurs, employés, actionnaires-ont des préférences personnelles en matière environnementale ou sociale et qu'ils peuvent récompenser les entreprises s'engageant dans les directions qui leur importent. Les engagements pris par les entreprises en matière de RSE et d'AV servent alors à leur signaler la performance sociale ou environnementale d'une entreprise. De nombreuses contributions traitent du consumérisme vert et proposent des modèles de différenciation des produits (voir par exemple Arora et Gangopadhyay, 1995; Besley et Ghatak, 2007). Brekke et Nyborg (2004) s'intéressent au marché du travail et montrent que la RSE peut permettre d'attirer des employés motivés, et donc plus productifs.

Le point de départ de la seconde branche de la littérature est différent. Améliorer la performance sociale ou environnementale est a priori coûteux. La RSE ou les AV n'émergent alors à l'équilibre que s'ils sont moins coûteux pour la firme que les politiques de régulation qui seraient mises en place en leur absence. Certains articles s'intéressent aux AV (Manzini et Mariotti, 2003; Segerson et Miceli, 1998; Glachant, 2007), d'autres aux formes unilatérales que sont l'auto-régulation ou à la RSE (Maxwell et alii, 2000; Heyes, 2005). 
Notre travail est plus proche de celui de Lyon et Maxwell (2003) qui analysent la RSE et (une forme $\left.d^{\prime}\right) A V$ dans un cadre unifié. La séquence décrite par leur modèle est la suivante. Après une étape de RSE, le régulateur initie un processus législatif, qui aboutit à un quota avec une certaine probabilité fixe. En dernière étape, un AV est mis en place si la tentative de légiférer n'a pas abouti. Une hypothèse clef est que l'AV est associé à une subvention qui fournit à la firme les incitations à s'engager. La première étape de notre modèle est identique mais les deux suivantes sont inversées : le régulateur tente d'obtenir un accord volontaire à la deuxième étape et ne s'engage dans la voie législative qu'en troisième étape si la firme refuse l'accord. Dans notre modèle, la firme s'engage donc pour éviter ou atténuer le quota déterminé par voie législative.

Notre séquence nous semble plus réaliste. Dans la pratique, les AV ne conduisent pas à des transferts monétaires vers les entreprises. Lyon et Maxwell (2003) en conviennent d'ailleurs. Mais ils justifient leur hypothèse en soulignant qu'ils sont souvent associés à une assistance technique ou à d'autres d'avantages non pécuniaires de la part de la puissance publique. Il nous semble que, dans la réalité, ces bénéfices sont trop modestes pour influencer significativement le comportement des firmes. A partir du moment où l'AV n'est pas associé à une subvention, la séquence que nous modélisons -négocier d'abord et légiférer en cas de désaccord-est la seule permettant l'émergence d'un accord à l'équilibre.

Nous montrons que l'effort de RSE réduit le bien-être social par rapport à un scénario sans RSE quand la séquence est RSE $\rightarrow \mathrm{AV} \rightarrow$ législation. Ce résultat diffère de ceux de Lyon et Maxwell (2003). En revanche, nous montrons que la RSE augmente le bien être social en l'absence d'AV, c'est-à-dire quand la séquence se limite à : RSE $\rightarrow$ législation. La RSE et les AV ne sont donc pas complémentaires dans l'optique d'un régulateur. Faut-il alors renoncer aux accords volontaires quand les firmes font déjà de la RSE ? La réponse est en fait ambiguë et dépend de la répartition du pouvoir de négociation entre la firme et le régulateur. En présence de RSE, les AV ne restent utiles que quand le régulateur dispose d'un fort pouvoir de négociation. Ainsi, l'usage de solutions négociées dans les politiques publiques, $\mathrm{s}^{\prime} \mathrm{il}$ apparaît Pareto-améliorant tant que le comportement des entreprises est fixé, s'avère moins efficace, voire néfaste, quand les entreprises peuvent préempter ces négociations.

L'article comporte quatre parties. Dans la section 2, nous présentons la version du modèle avec la séquence complète RSE $\rightarrow$ AV $\rightarrow$ législation. Nous étudions l'équilibre dans la section 3. Ensuite, nous nous interrogeons dans la section 4 sur l'intérêt d'interdire 
les AV en analysant une séquence RSE $\rightarrow$ législation. Enfin, la conclusion rassemble les principaux résultats.

\section{Modèle}

Nous considérons un jeu séquentiel entre une firme ${ }^{4}$ et un régulateur dans lequel $q$ est la variable pertinente. Nous l'assimilerons dans la suite à un niveau de dépollution. Mais les mécanismes sont en tout point semblables si $q$ décrit l'impact social ou la qualité des conditions de travail, deux dimensions souvent associées à la RSE.

\subsection{Paiements bruts}

Le coût d'abattement de la firme est $C(q)$, et il vérifie $C(0)=C^{\prime}(0)=0, C^{\prime}, C^{\prime \prime}>0$. Nous supposons que le régulateur cherche à maximiser le bien être social. Le bénéfice environnemental lié à l'abattement est $B(q)$, et supposé concave. Ainsi, le bien-être (brut des coûts éventuels de régulation) est

$$
W(q)=B(q)-C(q)
$$

C'est une fonction strictement concave et nous noterons $q^{*}$ le niveau optimal d'abattement défini implicitement par $W^{\prime}\left(q^{*}\right)=0$. Nous supposons naturellement $W^{\prime}(0)>0$, et sans perte de généralité, nous utilisons la normalisation $W(0)=0$.

\subsection{La législation}

Le régulateur ne peut adopter lui-même les lois. C'est la responsabilité du corps législatif.Il peut seulement initier le processus. Nous supposons qu'il propose au parlement l'adoption d'un quota prescrivant un niveau minimal d'abattement $L^{5}$. Mais des imperfections politiques, un temps limité pour les débats législatifs, des priorités politiques et des pressions des entreprises, empêche l'adoption systématique de sa proposition.

Plus spécifiquement, nous supposons que le quota est adopté avec une probabilité $p<1$. Le régulateur peut faire des efforts pour augmenter cette probabilité. Il peut par exemple produire des études pour convaincre les législateurs que la nouvelle législation ne pénaliserait pas trop la compétitivité de la firme, que le type de pollution est véritablement nocive, etc. Formellement, nous supposons que l'adoption avec une probabilité $p$ coûte $\gamma(p)$ au régulateur, avec $\gamma(0)=\gamma^{\prime}(0)=0$ et $\gamma^{\prime}, \gamma^{\prime \prime}>0$. Le fait que la probabilité 
soit nulle si aucun effort n'est fait est une normalisation sans incidence sur les résultats. Poser un coût marginal nul pour un effort nul permet de toujours obtenir des solutions intérieures. Enfin, nous supposons $\gamma^{\prime \prime \prime} \geq 0$ pour des raisons de confort technique.

Faire l'hypothèse que la voie législative est risquée est à la fois réaliste ${ }^{6}$ et usuel dans la littérature sur les accords volontaires ${ }^{7}$. Toute aussi réaliste, $1^{\prime}$ hypothèse que le régulateur peut faire des efforts pour accroître cette probabilité est en revanche originale. ${ }^{8}$ Dans les articles cités dans l'introduction, $p$ est exogène (Segerson et Miceli, 1998) ou influencée par des groupes de pression. ${ }^{9}$. Lyon et Maxwell (2003) font l'hypothèse que $p$ décroît avec la sévérité de la législation. ${ }^{10}$

\subsection{Déroulement du jeu}

Le jeu comprend les trois étapes déjà présentées :

1. (Responsabilité Sociale de l'Entreprise) La firme s'engage de manière unilatérale et irréversible sur un niveau d'abattement minimal $r$.

2. (Accord Volontaire) Le régulateur et la firme négocient un niveau d'abattement, $q^{A V}$. Si les deux parties s'accordent, la firme, la firme respecte l'accord.

3. (Législation) En cas de désaccord, le régulateur initie le processus législatif en choisissant le quota $L$ et la probabilité $p$, au coût $\gamma(p)$. Avec une probabilité $p$, la nouvelle législation est adoptée et la firme réduit ses émissions pour se mettre en conformité. Sinon, l'abattement reste au niveau $r$.

Remarquons que, dans cette séquence, le processus continue quel que soit le niveau de RSE choisi en première étape. En revanche, il s'arrête en seconde étape en cas d'accord volontaire. C'est la différence clef entre la RSE et les AV dans notre modèle.

\section{Analyse de l'équilibre}

\subsection{Législation}

Nous résolvons le jeu à rebours en commençant par l'étape législative. Supposons donc que l'entreprise s'est engagée sur un certain niveau $r$ de dépollution lors de la première étape de RSE . étant donné nos hypothèse, le régulateur va proposer le quota $L=q^{*}$ puisque la sévérité de l'abattement n'a pas d'influence sur $p .{ }^{11}$ L'effort législatif pour 
convaincre les législateurs est alors défini par :

$$
p(r) \equiv \underset{p}{\operatorname{argmax}} p W\left(q^{*}\right)+(1-p) W(r)-\gamma(p)
$$

Pour tout niveau $r$ donné, la probabilité optimale est unique du fait de la stricte concavité de l'objectif. Nous pouvons déjà remarquer :

Lemme 1 La probabilité de succès de l'action législative décroît avec le niveau de la RSE : $p^{\prime}(r)<$ 0 .

Preuve. La condition du premier-ordre $\gamma^{\prime}(p(r))=W\left(q^{*}\right)-W(r)$ tient nécessairement, sinon on aurait $r>q^{*}$, ce qui est strictement dominé pour la firme. La différentielle totale de la condition du premier ordre par rapport à $r$ donne $p^{\prime}(r)=-W^{\prime}(r) / \gamma^{\prime \prime}(p(r))$ qui est négatif puisque $r \leq q^{*}$.

C'est le mécanisme fondamental de notre modèle : la RSE permet de diminuer l'effort du législateur. En effet, il a moins à gagner par la voie législative puisqu'un certain niveau d'abattement est d'ores et déjà déjà acquis.

\subsection{Accord Volontaire}

La négociation d'un objectif pour l'accord volontaire, $q^{A V}$, a lieu sous les contraintes de participation suivantes :

$$
\begin{gathered}
W\left(q^{A V}\right) \geq p(r) W\left(q^{*}\right)+(1-p(r)) W(r)-\gamma(p(r)) \equiv \bar{U}(r) \\
C\left(q^{A V}\right) \leq p(r) C\left(q^{*}\right)+(1-p(r)) C(r) \equiv \bar{C}(r)
\end{gathered}
$$

qui signifient que le régulateur obtient plus dans l'accord volontaire que la valeur espérée de la législation moins son effort législatif, d'une part, et que le coût d'abattement pour la firme dans l'accord volontaire est inférieur à l'espérance de son coût en cas de tentative de législation, d'autre part. Les paiements de réservation paramétrés par l'investissement RSE consenti lors de la première étape sont ainsi notés $\bar{U}(r)$ et $\bar{C}(r)$.

Comme $W$ et $C$ sont respectivement concave et convexe, $1^{\prime}$ ensemble des $q^{A V}$ admissibles est non vide. Par exemple, $q^{A V}=p(r) q^{*}+(1-p(r)) r$ satisfait strictement les deux contraintes. De plus, comme les deux contraintes sont continues, l'ensemble admissible est un intervalle de la forme $\left[\underline{q}^{A V}, \bar{q}^{A V}\right]$ dont la borne inférieure correspond à une contrainte de participation saturée pour le régulateur et la borne supérieure à une contrainte de participation saturée pour la firme. On peut donc écrire : 
Lemme 2 Pour tout $r$ donné, un AV émerge à l'équilibre et il est Pareto-supérieur à la voie législative.

Ce lemme réplique un résultat classique de la littérature sur les AV. Le message est le suivant : lorsqu'un AV émerge à l'équilibre, il est moins contraignant que la législation optimale $\left(q^{A V}<q^{*}\right)$. Mais il est préféré par le régulateur. Une première raison est la concavité d'au moins une des fonctions d'utilité qui confère un avantage au AV dont le résultat est certain alors que la voie législative qui est incertaine. La deuxième raison est qu'un AV lui permet d'économiser le coût législatif $\gamma(p)$.

Dans la suite, il s'avèrera utile de pouvoir travailler avec un seul niveau d'équilibre pour l'AV, plutôt que l'ensemble des niveaux d'abattement admissibles $\left[q^{A V}, \bar{q}^{A V}\right]$. Ce niveau dépend évidemment de l'allocation du pouvoir de négociation entre le régulateur et la firme. Pour paramétrer la frontière de Pareto, nous allons considérer la solution de Nash généralisée ${ }^{12}$ avec des pouvoirs de négociations $\alpha$ pour le régulateur et $1-\alpha$ pour la firme $(0 \leq \alpha \leq 1)$. Le programme de Nash s'écrit alors :

$$
\max _{q}[W(q)-\bar{U}(r)]^{\alpha}[\bar{C}(r)-C(q)]^{1-\alpha}
$$

et la solution de ce programme, notée $q^{A V}(\alpha, r)$, est donnée implicitement par la condition du premier ordre :

$$
\left(\frac{\alpha}{1-\alpha}\right) \frac{W^{\prime}\left(q^{A V}(\alpha, r)\right)}{C^{\prime}\left(q^{A V}(\alpha, r)\right)}=\frac{\Delta W(\alpha, r)}{\Delta C(\alpha, r)}
$$

où l'on utilise les notations :

$$
\Delta W(\alpha, r) \equiv W\left(q^{A V}(\alpha, r)\right)-\bar{U}(r) \quad \text { et } \quad \Delta C(\alpha, r) \equiv \bar{C}(r)-C\left(q^{A V}(\alpha, r)\right)
$$

\subsection{RSE}

Nous avons vu que l'entreprise et le régulateur arrivent toujours à un accord à l'étape 2. Quand la firme choisit son niveau de RSE à la première étape, elle résout donc :

$$
\min _{r} C\left(q^{A V}(\alpha, r)\right)
$$

où $q^{A V}(\alpha, r)$ est donné par (3). Nous utiliserons l'indice 1 pour désigner la solution de ce programme.

Nous pouvons maintenant établir un résultat central de l'article. Si la firme entreprend des activités de RSE à l'équilibre (c'est-à-dire si $r_{1}>0$ ), on a nécessairement 
$C\left(q^{A V}(\alpha, 0)\right)>C\left(q^{A V}\left(\alpha, r_{1}\right)\right)$, et donc $\left.q^{A V}(\alpha, 0)\right)>q^{A V}\left(\alpha, r_{1}\right)$. En d'autres termes, la firme n'entreprend des activités de RSE que si le niveau de l'AV s'en trouve diminué. Cela implique immédiatement un bien-être réduit puisque $W$ est une fonction croissante de $q^{A V}$ avant $q^{*}$. En résumé :

Proposition 1 Si la firme entreprend des activités de RSE à l'équilibre $\left(r_{1}>0\right)$, le bien-être social s'en trouve diminué.

La RSE a donc un impact négatif. Mais se produit-elle à l'équilibre? Pour répondre à cette question, il faut résoudre le programme de la firme (4). Nous obtenons :

Proposition 2 Il existe une valeur du pouvoir de négociation du régulateur, $\hat{\alpha} \in(0,1)$, telle que la firme entreprend des actions de RSE $\left(r_{1}>0\right)$ si et seulement si $\alpha>\hat{\alpha}$.

Preuve. Voir en Annexe.

L'intuition de ce résultat est assez simple. Quand le pouvoir de négociation de l'autorité publique est limité $(\alpha \leq \hat{\alpha})$, l'entreprise n'a pas intérêt à préempter la régulation puisqu'elle s'approprie une part importante du surplus dans l'accord négocié. Au contraire, face à un régulateur âpre à la négociation $(\alpha>\hat{\alpha})$, l'entreprise préfère réduire le surplus en jeu dans la négociation à venir où elle sera perdante et rendre le régulateur moins agressif en augmentant son point de menace.

Les résultats de la proposition 1 et de la proposition 2 pourraient suggérer que le pouvoir de négociation a un effet ambigu sur le bien-être. En effet, un pouvoir de négociation élevé aide le régulateur a obtenir un accord volontaire plus ambitieux; mais cela incite la firme à préempter la régulation lors de la première étape. En fait, le premier effet l'emporte :

Lemme 3 Le niveau d'abattement d'équilibre, et donc le bien-être, augmentent avec le pouvoir de négociation du régulateur.

Preuve. Voir en Annexe.

Quelles sont les implications en termes de politiques publiques des résultats obtenus jusque là? Nous avons vu que la RSE et les AV n'étaient pas complémentaires du point de vue de la puissance publique puisque la RSE réduit l'ambition de l'AV. Est-il possible de résoudre ce problème? Deux solutions viennent à l'esprit. La première consisterait à 
interdire les activités de RSE et à continuer à utiliser les accords volontaires. Cela semble difficilement envisageable dans une économie de marché. La seconde option serait d'interdire au régulateur de négocier des accords avec les entreprises, ce qui semble légalement plus facile. Remarquons toutefois que cette solution demande un engagement fort ex ante puisque, une fois la deuxième étape atteinte, signer un AV est toujours Paretooptimal.

Pour explorer l'intérêt de cette solution, nous étudions maintenant un jeu sans AV.

\section{Un jeu sans AV}

Nous supprimons simplement la deuxième étape du jeu. L'étape législative est identique à celle analysée dans le lemme 1 . Il suffit donc de résoudre la première étape du jeu.

\subsection{RSE}

Nous noterons $r_{2}$ le niveau d'équilibre dans ce scenario. La firme minimise son coût d'abattement espéré : ${ }^{13}$

$$
\min _{r}\left\{p(r) C\left(q^{*}\right)+(1-p(r)) C(r)\right\}
$$

Il est ensuite facile d'obtenir :

Proposition 3 En l'absence d'accord volontaire, la firme s'engage toujours dans des activités de $R S E$ : il existe un unique équilibre, avec $r_{2}>0$.

Preuve. Pour $r=0$, on a :

$$
\bar{C}^{\prime}(0)=p^{\prime}(0) C\left(q^{*}\right)<0
$$

Donc le minimum $r_{2}$ est strictement positif. De plus,

$$
\bar{C}^{\prime \prime}(r)=p^{\prime \prime}(r)\left(C\left(q^{*}\right)-C(r)\right)-2 C^{\prime}(r) p^{\prime}(r)+(1-p(r)) C^{\prime \prime}(r)
$$

Par le lemme 1, nous savons $p^{\prime}(r)=-W^{\prime}(r) / \gamma^{\prime \prime}(r)<0$ à l'équilibre pour tout $r$. On obtient en dérivant :

$$
p^{\prime \prime}(r) \gamma^{\prime \prime}(p(r))=p^{\prime}(r)^{2} \gamma^{\prime \prime \prime}(p(r))-W^{\prime \prime}(r)
$$


dont le membre de droite est positif puisque $\gamma^{\prime \prime \prime} \geq 0$ et $W$ est concave. Cela implique $p^{\prime \prime}>0$. Donc $\bar{C}$ est convexe et admet un unique minimum.

La firme préfère maintenant préempter la législation alors que, dans le cas précédent, la RSE n'apparaissait qu'en présence d'un fort pouvoir de négociation du régulateur.

Dans le lemme 1, les investissements en RSE réduisaient la probabilité de législation $p$. Faut-il en conclure que le bien-être serait supérieur sans RSE ? Là encore, le résultat est différent du cas avec AV :

Proposition 4 En l'absence d'accord volontaire, la RSE est socialement bénéfique et le bien-être augmente avec $r_{2}$.

Preuve. Pour tout $r$, Le régulateur atteint un paiement $\bar{U}(r)=p(r) W\left(q^{*}\right)+(1-$ $p(r)) W(r)-\gamma(p(r))$. On obtient en dérivant:

$$
\bar{U}^{\prime}(r)=p^{\prime}(r)\left[W\left(q^{*}\right)-W(r)-\gamma^{\prime}(p(r))\right]+(1-p(r)) W^{\prime}(r) .
$$

Par le lemme 1, nous savons que $\gamma^{\prime}(p)=W\left(q^{*}\right)-W(r)$. Ainsi, $\bar{U}^{\prime}(r)=(1-p(r)) W^{\prime}(r)$, qui est positif $\left(W^{\prime}(r)>0\right.$ puisque $\left.r<q^{*}\right)$.

Dans ce scénario, la RSE est socialement utile et son usage doit être promu sans restriction. Le régulateur ne pâtit pas de la RSE dans ce contexte. En l'absence de RSE, le régulateur choisirait un certain niveau d'effort. S'il choisit le même niveau quand la firme investit en RSE à la première étape, son gain ne peut qu'être que supérieur puisque que le gain est identique sur tous les plans sauf dans le cas où la législation n'est pas adoptée. Mais, dans ce cas, l'abattement final est $r_{2}$, ce qui est préférable que l'absence d'abattement dans le scénario sans RSE.

\subsection{Faut-il renoncer aux Accords Volontaires?}

Comme nous l'avons vu, la firme choisit systématiquement d'entreprendre une politique de RSE en l'absence d'AV ce qui est conforme à l'intérêt général dans ce contexte. Doit-on pour autant interdire les AV? Nous comparons dans cette section les résultats des deux alternatives.

Nous nous concentrons d'abord sur le cas d'un régulateur sans pouvoir de négociation, $\alpha=0$. Dans le scenario où les AV sont autorisés, la contrainte de participation du 
régulateur (1) est alors saturée, $W\left(q^{A V}\right)=\bar{U}(r)$, de telle sorte que l'AV d'équilibre est tel que :

$$
W\left(q^{A V}\right)=p\left(r^{1}\right) W\left(q^{*}\right)+\left(1-p\left(r^{1}\right)\right) W\left(r^{1}\right)-\gamma\left(p\left(r^{1}\right)\right)
$$

Ainsi, l'AV et la législation donnent dans ce cas le même niveau de bien-être net.

Dans le scenario sans AV, le bien-être net est formellement similaire :

$$
\bar{U}\left(r_{2}\right)=p\left(r_{2}\right) W\left(q^{*}\right)+\left(1-p\left(r_{2}\right)\right) W\left(r_{2}\right)-\gamma\left(p\left(r_{2}\right)\right)
$$

sauf que les niveaux de RSE sont différents. La proposition 2 indique que $r_{1}=0$ quand $\alpha=0$, tandis que la proposition 3 indique que $r_{2}>0$ quand les $\mathrm{AV}$ sont interdits. Puisque les AV augmentent le bien-être net dans ce cas (proposition 4), nous en déduisons immédiatement que l'interdiction des AV est socialement préférable.

Dans l'autre cas extrême, $\alpha=1$, nous avons

$$
C\left(q^{A V}\right)=p\left(r_{1}\right) C\left(q^{*}\right)+\left(1-p\left(r_{1}\right)\right) C\left(r_{1}\right)
$$

avec $r_{1}>0$. L'AV et la législation sont maintenant équivalents en coûts pour la firme. Le niveau de RSE est donc le même dans le scénario législatif que dans celui de l'AV: $r_{1}=r_{2}$. De plus, la contrainte de participation du régulateur n'est pas saturée ce qui signifie :

$$
W\left(q^{A V}\right)>p\left(r_{1}\right) W\left(q^{*}\right)+\left(1-p\left(r_{1}\right)\right) W\left(r_{1}\right)-\gamma\left(p\left(r_{1}\right)\right)
$$

Puisque $r_{1}=r_{2}$, le terme de droite est également le bien-être net à l'équilibre. L'utilisation des AV est donc ici préférable.

Dans les cas intermédiaires, c'est-à-dire $\alpha \in(0,1)$, le lemme 3 indique que le bien-être avec $\mathrm{AV}$ est croissant en $\alpha$, tandis qu'il ne dépend pas du pouvoir de négociation dans le scenario sans AV. Ainsi, il existe une unique valeur seuil de $\alpha$ telle que l'interdiction des AV est souhaitable pour tout pouvoir de négociation inférieur. Nous rassemblons ces observations dans la proposition suivante :

Proposition 5 Il existe une valeur seuil du pouvoir de négociation du régulateur $\tilde{\alpha} \in(0,1)$ telle que l'interdiction de l'usage d'AV améliore (détériore) le bien-être social si $\alpha \leq \tilde{\alpha}(\alpha>\tilde{\alpha})$.

Cette proposition fait écho à la distinction usuelle entre les programmes publics volontaires et les accords volontaires négociés évoquée dans l'introduction. Ce sont deux types d'AV qui, par construction, allouent différemment le pouvoir de négociation entre la puissance publique et le secteur privé. Dans un programme volontaire, le régulateur 


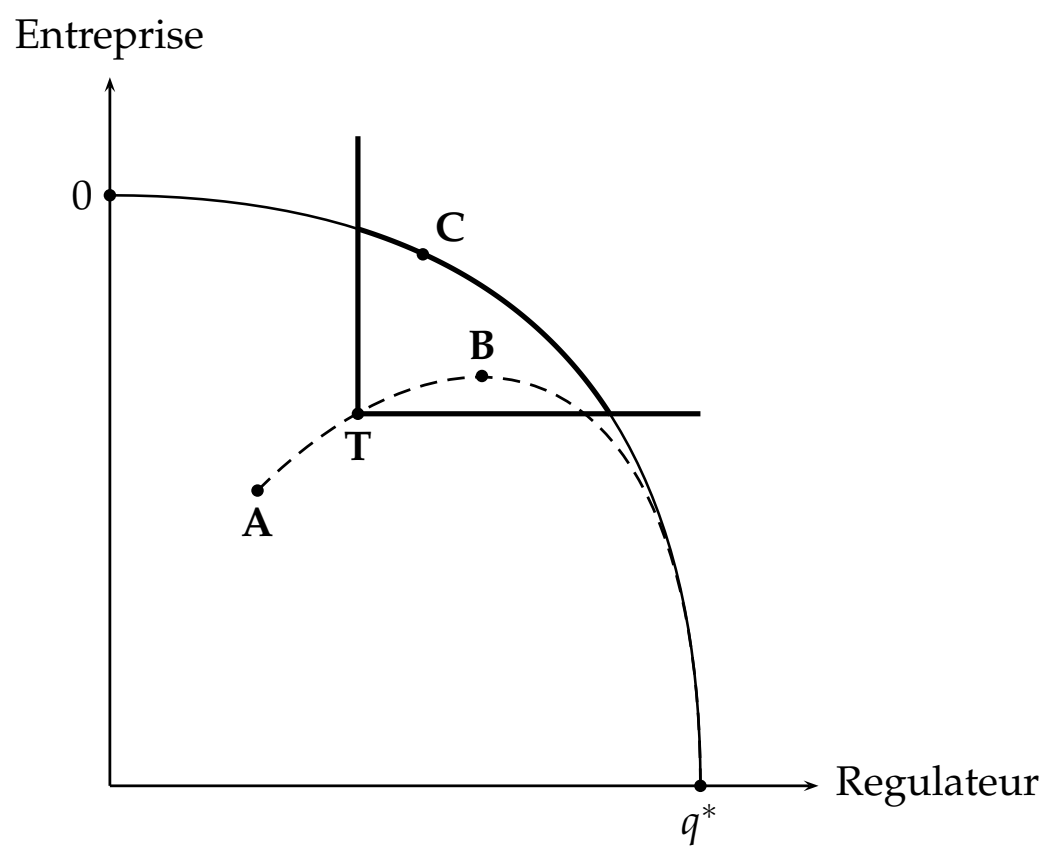

FIGURE 1 - Equilibre dans les différentes séquences

propose des objectifs environnementaux que la firme est libre d'accepter ou de refuser. Il n'y a pas de négociation entre les parties pour définir ces objectifs. Ainsi, un programme volontaire donne tout le pouvoir au régulateur qui fait une offre à prendre ou à laisser. Les objectifs environnementaux des accords négociés sont déterminés de façon conjointe par le régulateur et les entreprises visées. La proposition 5 suggère que les programmes volontaires sont plus adaptés quand les firmes s'engagent dans la RSE.

\subsection{Bilan}

La figure 1 présente les équilibres selon l'alternative choisie. Le point A correspond au cas où seule la législation est utilisée et où la firme n'entreprend pas d'action de RSE. Dans ce cas, $r=0$, et le régulateur contrôle à travers son effort la loterie entre le point $q=0$, quand la législation échoue, et $q=q^{*}$, quand la législation est mise en place.

La courbe en pointillés correspond à l'ensemble des gains atteints quand $r$ varie entre 0 (point $\mathrm{A}$ ) et $q^{*}$. Le point $\mathrm{B}$ correspond à l'équilibre atteint dans le scénario sans $\mathrm{AV}$. Il est clair qu'entre A et B, un accroissement de $r$ est Pareto-améliorant. Ainsi, la firme entreprend des investissements en RSE tant que le point B n'est pas atteint et la RSE améliore le bien-être (proposition 4). 
Enfin, le point $\mathrm{C}$ correspond à l'AV d'équilibre pour un pouvoir de négociation $\alpha<\tilde{\alpha}$. Le point $\mathrm{T}$ est le point de menace dans l'AV quand la firme a choisi un certain niveau (d'équilibre) de RSE en première période. Les paiements du status quo de la négociation sont les gains espérés si la route législative est choisie. Les droites partant du point $T$ représente les contraintes de participation du régulateur (verticale) et de l'entreprise (horizontale). On peut mentionner deux cas extrêmes : si $\alpha=1$, pour tout niveau de RSE, l'issue de la négociation est sur la droite horizontale pour tout $r$, et la firme choisit donc $r$ au même niveau que dans le cas sans AV. A l'autre extrême, $\alpha=0$, l'issue de la négociation est sur la ligne verticale, et la firme n'a aucun intérêt à s'engager dans la RSE de telle sorte qu'à l'équilibre $r=0$ (voir proposition 2 et lemme 3). L'équilibre $C$ correspond ainsi à une situation dans laquelle le régulateur préférerait l'équilibre B (proposition 5).

\section{Conclusion}

Le modèle proposé dans cet article décrit un jeu dans lequel un régulateur environnemental doit consentir un effort pour que la régulation optimale soit effectivement votée par le parlement. Concrètement, il doit préparer un dossier, réaliser des études pour convaincre les législateurs que la proposition de loi améliorerait le bien-être, etc. Dans ce contexte, le temps et les ressources qu'il va consacrer à cette activité dépend de l'écart endogène entre le statu quo et la situation après législation. Les actions de RSE unilatérales peuvent alors être instrumentalisées par les entreprises pour réduire les incitations du régulateur à faire ces efforts.

Notre analyse montre que la RSE utilisée stratégiquement par la firme améliore malgré tout le bien-être social quand le régulateur choisit la voie législative. En revanche, elle dégrade le bien-être si le régulateur a recours à des AV. L'intuition générale est que la RSE permet à la firme de réduire la menace législative qui va déterminer la sévérité de l'AV.

Ces résultats suggèrent donc que la RSE et les AV ne sont pas complémentaires du point de vue de l'intérêt général. Comme il n'est pas possible d'empêcher les firmes de pratiquer la RSE, doit-on interdire l'usage des AV? Nous montrons que la pertinence de cette interdiction dépend du pouvoir de négociation du régulateur. Lorsqu'il est important, l'impact négatif de la RSE est plus que compensé par les gains de bien-être obtenus en signant un AV. Pour un pouvoir de négociation faible, il est en revanche optimal pour le régulateur de s'interdire de négocier des $\mathrm{AV}$. 
Contrairement à un sentiment répandu, la RSE n'est donc pas toujours socialement bénéfique, en particulier dans les secteurs où les accords volontaires peuvent être utilisée. Ces résultats indiquent également que les programmes volontaires fondés sur des engagements formulés par la puissance publique "à prendre ou à laisser" sont plus adaptés dans les secteurs où la RSE est déjà développée. En effet, ces programmes, bien que rigides, donnent par construction le pouvoir de négociation au régulateur.

Notre modèle reste très simple. Plusieurs aspects appellent de futurs développements, particulièrement sur le contenu informationnel de la RSE et son rôle de signal (Denicolo, 2000; Fisman et alii, 2006), et sur la crédibilité de l'engagement des firmes (Glachant, 2007). 


\section{Notes}

${ }^{1}$ L'Allemagne et le Japon les ont même utilisés dès les années 70.

${ }^{2}$ En particulier, nous supposons que l'engagement est aussi fort pour l'entreprise dans la RSE et dans les accords volontaires et que la fonction de coût est identique selon que l'abattement est réalisé dans une phase de RSE ou suite à un AV. Ceci nous permet de nous concentrer exclusivement sur le jeu d'influence mené par la firme quand elle choisit son niveau de RSE, sans donner d'avantages ad hoc à l'une ou l'autre des situations.

${ }^{3}$ Voir Lyon et Maxwell (2002) et Lyon et Maxwell (2007) pour une revue détaillée de la littérature.

${ }^{4}$ En réalité, des entreprises se regroupent souvent pour signer des accords volontaires sectoriels. Notre modèle s'applique directement à ce cas quand les membres de la coalition ont résolu leur problème d'action collective. Cette hypothèse est habituelle dans la littérature sur les accords volontaires.

${ }^{5}$ L'instrument utilisé à ce stade-quota, norme, taxe, etc.-n'a pas d'importance dans l'analyse.

${ }^{6}$ A titre d'exemple, en France pendant la période 1997-2002, le gouvernement a fait 476 propositions de loi, parmi lesquelles 351 ont été finalement adoptées par le Parlement, ce qui implique une probabilité moyenne d'adoption égale à 0,74 .

${ }^{7}$ Glachant (2005); Lyon et Maxwell (2003); Heyes (2005); Segerson et Miceli (1998); Manzini et Mariotti (2003) utilisent tous cette hypothèse.

${ }^{8}$ Voir Maxwell et Decker (2006) pour un cas où faire respecter une législation en place est coûteux. Dans leur modèle, la RSE peut être utilisée pour faciliter les coûts de respect de législations existantes. Dans ce cas, la RSE est utilisée comme un substitut partiel de l'effort de contrôle du régulateur.

${ }^{9}$ L'activité des groupes de pression peut être modélisée explicitement comme dans Glachant (2005) sans changer nos résultats.

${ }^{10}$ Dans une version antérieure, nous étudions ces deux alternatives. Nous montrons qu'introduire des groupes de pressions qui influent sur la probabilité d'adoption conduit formellement à un coût pour le législateur ayant exactement les propriétés de la fonction $\gamma$. Nous étudions aussi la dépendance de $p$ au niveau de sévérité du quota et montrons que l'effet préemptif que nous étudions est préservé.

${ }^{11}$ Cette hypothèse n'est pas cruciale. On obtiendrait des résultats qualitativement identiques en considérant que la probabilité d'adoption est affectée négativement par le niveau du quota proposé.

${ }^{12}$ Nous aurions pu considérer aussi que l'un des joueurs fait une proposition avec une probabilité donnée, décrivant le pouvoir de négociation. Cependant, un tel modèle de négociation ne serait pas optimal en termes de risque, étant donné la stricte convexité de la frontière de Pareto.

${ }^{13} \mathrm{Ce}$ niveau correspond exactement à son utilité de réservation dans $\mathrm{l}^{\prime} \mathrm{AV}, \overline{\mathrm{C}}(r)$ analysé précédemment. 


\section{Bibliographie}

Arora, S. et Gangopadhyay, S., 1995. Toward a theoretical model of voluntary overcompliance, Journal of Economic Behavior and Organization, vol. 28, pp. 289-309.

Baron, D.P. 2008. Managerial contracting and corporate social responsibility, Journal of Public Economics, vol. 92, pp. 268-288.

Besley, T. et Ghatak M., 2007. Retailing public goods : the economics of corporate social responsibility, Journal of Public Economics, vol. 91, pp. 1645-1663.

Brekke, K. et Nyborg, K., 2004. Moral hazard and moral motivation : Corporate social responsibility as labor market screening, Mimeo, Department of Economics, University of Oslo.

Denicolo, V., 2000. A Signalling Model of Environmental Overcompliance, FEEM Nota di Lavoro 77.2000.

Glachant, M., 2005. Voluntary Agreements in a Rent Seeking Environment, in E. Croci (Ed.) Handbook of Environmental Voluntary Agreements, Dordrecht, Holland, Kluwer Academic Publishers, pp. 49-67.

Glachant, M., 2007. Non-binding Voluntary Agreements, Journal of Environmental Economics and Management, vol. 54, pp. 32-48.

Fisman, R., Heal, G. et Nair, V., 2006. A Model of Corporate Philanthropy, mimeo Columbia Business School.

Heyes, A., 2005. A Signaling-motive for Self-regulation in the Shadow of Coercion, Journal of Business and Economics, vol. 57, pp. 238-246.

Lyon, T. et Maxwell J., 2002. Voluntary Approaches to Environmental Regulation : A Survey, in M. Franzini and A. Nicita (eds.), Economic Institutions and Environmental Policy : Past, Present and Future, Aldershot, Hampshire, UK : Ashgate Publishing Ltd.

Lyon, T. et Maxwell, J., 2003. Self-regulation, taxation and public voluntary environmental agreements, Journal of Public Economics, vol. 87, pp. 1453-1486.

Lyon, T. et Maxwell J., 2007. Corporate Social Responsibility and the Environment : A Theoretical Perspective, Mimeo. Kelley School of Business, Indiana University, Bloomington. 
Lutz, S., Lyon, T. et Maxwell, J., 2000. Quality Leadership When Regulatory Standards Are Forthcoming, Journal of Industrial Economics, vol. 48, pp. 331-348.

Maxwell, J. et Decker, C., 2006. Voluntary Environmental Investment and Responsive Regulation, Environmental and Resource Economics, vol. 33, pp. 425-439.

Maxwell, J. et Lyon, T., Hackett, S., 2000. Self regulation and social welfare : the political economy of corporate environmentalism, Journal of Law and Economics, vol. 43, pp. 583-617.

Manzini, P. et Mariotti, M., 2003. A bargaining model of voluntary environmental agreements, Journal of Public Economics, vol. 87, pp. 2725-2736.

Segerson, K. et Miceli, T., 1998. Voluntary Environmental Agreements : Good or Bad News for Environmental Protection? Journal of Environmental Economics and Management, vol. 36, pp. 109-130. 


\section{A Annexe}

\section{A.1 Preuve de la proposition 2}

Le programme (4) équivaut à $\operatorname{Min}_{r} q^{A V}(\alpha, r)$. Nous le résolvons comme suit : 1$)$ nous calculons $\partial q^{A V} / \partial r$ pour $\left.r=0.2\right)$ Nous montrons que $\partial q^{A V}(\alpha, 0) / \partial r$ décroît avec $\alpha$ sur $[0,1]$. 3) Nous montrons que $\partial q^{A V}(0,0) / \partial r>0$, de telle sorte que $r_{1}=0$ quand $\alpha=0$. 4) Nous montrons que $\partial q^{A V}(1,0) / \partial r<0$, ce qui implique l'existence d'un $\hat{\alpha}<1$ tel que $r_{1}>0$ pour $\alpha>\hat{\alpha}$.

1) En dérivant (3) et en réarrangeant, on obtient

$$
\frac{\partial q^{A V}}{\partial r}=\frac{\Delta C(\alpha, r)(1-p(r)) W^{\prime}(r)+\Delta W(\alpha, r)\left[p^{\prime}(r)\left(C\left(q^{*}\right)-C(r)\right)+(1-p(r)) C^{\prime}(r)\right]}{W^{\prime}\left(q^{A V}\right) \Delta C(\alpha, r)+\Delta W(\alpha, r) C^{\prime}\left(q^{A V}\right)-\alpha \Delta C(\alpha, r) \frac{W^{\prime \prime}\left(q^{A V}\right) C^{\prime}\left(q^{A V}\right)-W^{\prime}\left(q^{A V}\right) C^{\prime \prime}\left(q^{A V}\right)}{(1-\alpha)\left[C^{\prime}\left(q^{A V}\right)\right]^{2}}}
$$

Le dénominateur est strictement positif puisque $W^{\prime}, \Delta C(\alpha, r), \Delta W, C^{\prime}>0$ et $W^{\prime \prime}<0$. Il suffit donc d'étudier le signe du numérateur. Soit $\Omega$ cette expression dans le cas $r=0$. On a

$$
\Omega(\alpha)=\Delta C(\alpha, 0)(1-p(0)) W^{\prime}(0)+\Delta W(\alpha, 0) p^{\prime}(0) C\left(q^{*}\right)
$$

2) En dérivant $\Omega$ par rapport à $\alpha$ :

$$
\Omega^{\prime}(\alpha)=\frac{\partial q^{A V}}{\partial \alpha}\left[-C^{\prime}(\alpha, 0)(1-p(0)) W^{\prime}(0)+W^{\prime}(\alpha, 0) p^{\prime}(0) C\left(q^{*}\right)\right]
$$

Le terme entre crochets est négatif. De plus $\partial q^{A V} / \partial \alpha>0$. En effet, en dérivant (3) on voit :

$$
\frac{\partial q^{A V}}{\partial \alpha}=\frac{W^{\prime} \Delta C+C^{\prime} \Delta W}{W^{\prime} C^{\prime}-\alpha W^{\prime \prime} \Delta C+(1-\alpha) C^{\prime \prime} \Delta W}>0
$$

où toutes les fonctions sont évaluées à $(\alpha, r)$. Le fait que cette expression soit positive provient des faits suivants : toute solution du programme de Nash est telle que $q^{A V}(\alpha, r)<$ $q^{*}$, les fonctions $\Delta$ sont positives, enfin $W$ et $C$ sont respectivement concave et convexe.

Au total, $\partial q^{A V}(\alpha, 0) / \partial r$ décroît avec $\alpha$ sur $[0,1]$.

3) Quand $\alpha=0, \Delta W=0$ donc $\Omega(0)=\Delta C(0,0)(1-p(0)) W^{\prime}(0)>0$. Ainsi, $\partial q^{A V}(0,0) / \partial r>$ 0 .

4) Quand $\alpha=1, \Delta C=0$ et donc $\Omega(1)=\Delta W(\alpha, 0) p^{\prime}(0) C\left(q^{*}\right)<0$.

\section{A.2 Preuve du lemme 3}

Soit $r_{1}(\alpha)$ le niveau d'effort de RSE en fonction de $\alpha$. On a :

$$
\frac{d q^{A V}\left(\alpha, r_{1}(\alpha)\right)}{d \alpha}=\frac{\partial q^{A V}\left(\alpha, r_{1}(\alpha)\right)}{\partial \alpha}+\frac{\partial r_{1}(\alpha)}{\partial \alpha} \frac{\partial q^{A V}\left(\alpha, r_{1}(\alpha)\right)}{\partial r}
$$


Nous avons obtenu dans la preuve de la proposition 2 que $\frac{\partial q^{A V}}{\partial \alpha}>0$. De plus, la proposition 2 indique que $r_{1}=0$ si $\alpha \leq \hat{\alpha}$, d'où $\partial r_{1}(\alpha) / \partial \alpha=0$. Ceci implique que $d q^{A V} / d \alpha=$ $\partial q^{A V} / \partial \alpha>0$. Quand $\alpha>\hat{\alpha}, r_{1}$ vérifie $\partial q^{A V}\left(\alpha, r_{1}(\alpha)\right) / \partial r=0$. Là encore, $d q^{A V} / d \alpha=$ $\partial q^{A V} / \partial \alpha>0$. 